Title:

Author(s):

\section{Submitted to:}

\section{Los Alamos}

\section{NATIONAL LABORATORY}

THE DBV STARS: PROGRESS AND PROBLEMS

P. A. Bradley

Whole Earth Telescope Workshop

(Special Issue of the Baltic Astronomical Journal) Ames, IA

July 1995

Los Alamos National Laboratory, an affirmative action/equal opportunity emplỏyer, is operated by the University of California for the U.S. Department of Energy under contract W-7405-ENG-36. By acceptance of this article, the publisher recognizes that the U.S. Government retains a nonexclusive, royalty-free license to publish or reproduce the published form of this contribution, or to allow others to do so, for U.S. Government purposes. The Los Alamos National Laboratory requests that the publisher identify this article as work performed under the auspices of the U.S. Department of Energy. 


\section{DISCLAIMER}

Portions of this document may be illegible in electronic image products. Images are produced from the best available original document. 
Baltic Astronomy, vol.4, $2 x x-x x x, 1995$.

\title{
THE DBV STARS: PROGRESS AND PROBLEMS
}

\author{
P.A. Bradley \\ XTA, MS B220, Los Alamos National Laboratory, Los Alamos, NM 87545
}

Received August 1, 1995.

Abstract. I describe the current status of our understanding of DBV white dwarf structure via asteroseismology, with an emphasis on what we learned through Whole Earth Telescope data.

Key words: Stars: white dwarfs, Stars: interiors, Stars: oscillations, Stars: individual: GD 358

\section{Introduction}

The DB white dwarfs are still something of a mystery, in spite of many years of study. They comprise the majority of the $20 \%$ or so of non-DA white dwarfs, and have effective temperatures between $11,000 \mathrm{~K}$ and $30,000 \mathrm{~K}$. The hottest DBs define the cool end of the so called "DB gap" that lies between $30,000 \mathrm{~K}$ and $45,000 \mathrm{~K}$; in this region, no helium atmosphere white dwarf is known. The existence of this gap presents a great puzzle concerning the origin and evolution of helium atmosphere white dwarfs. Asteroseismology of the DBV stars as a class will tell us what DBs just below the red edge of the DB gap are like. This, coupled with structural understanding of the pulsating PG 1159 stars, the interacting binary white dwarfs (IBWDs), and white dwarf evolution calculations should fill in the gaps of our knowledge about the DB white dwarfs and their origins.

Asteroseismology will give us total stellar mass and the helium layer mass determinations of the DB white dwarfs, which will help us decide what their progenitors are. Weidemann (1990) describes the most recent mean mass determinations for the $D B$ white dwarfs, and they are consistent with the mean mass of the DA white dwarfs determined by Bergeron, Saffer, \& Liebert (1992). However, there are

Send offprint requests to: P.A. Bradley

\section{DISCLAIMER}

This report was prepared as an account of work sponsored by an agency of the United States Government. Neither the United States Government nor any agency thereof, nor any of their employees, makes any warranty, express or implied, or assumes any legal liability or responsibility for the accuracy, completeness, or usefulness of any information, apparatus, product, or process disclosed, or represents that its use would not infringe privately owned rights. Reference herein to any specific commercial product, process, or service by trade name, trademark, manufacturer, or otherwise does not necessarily constitute or imply its endorsement, recommendation, or favoring by the United States Government or any agency thereof. The views and opinions of authors expressed herein do not necessarily state or reflect those of the United States Government or any agency thereof. 
no published studies done since the mid-1980's; the mean mass determination desperately needs to be redone with current models and observations. There are several theoretical and observational limits for the helium layer mass. First, the lack of helium fusion implies that the helium layer mass cannot be more than $\sim 10^{-2} \mathrm{M}_{\star}$. Pelletier et al. (1986) compare evolutionary diffusion models to observations of helium atmosphere white dwarfs "polluted" with carbon (the DQ white dwarfs). They duplicate the observed carbon abundances when the helium layer mass in their models is about $10^{-4} \mathrm{M}_{\star}$. Bradley \& Winget (1994) compare periods from theoretical models of DB white dwarfs to WET observations of GD 358 and derive a helium layer mass of $1.5 \times 10^{-6} \mathrm{M}_{\star}$. However, Dehner \& Kawaler (1995) show that the total amount of helium may be greater than Bradley \& Winget derive.

\section{Meet the DBV stars}

\section{General Remarks}

Our understanding of the DBV stars is affected by a brightness disparity which basically divides GD 358 from all the other DBVs. GD 358 is by far the brightest DBV star $\left(m_{b}=13.6\right)$, and is the best understood - in a relative sense - as a result. The next brightest DBV star is over two magnitudes fainter, meaning we would need $2.5 \mathrm{~m}$ telescopes to get the same signal-to-noise temporal spectroscopy, assuming everything else is equal. This presents a real problem for the WET in resolving the modal structure of the other DBV stars, and we are at a crossroads with the 1995 May run, with PG $1351+489$ as the primary target. We obtained the largest telescopes possible in the northern hemisphere for this faint DBV; if we cannot get satisfactory results here, then we may have to wait until we obtain more sensitivity from our instruments or we can piece together a network of telescopes 2 to 4 meters in diameter.

\section{The Stars}

Having said this, let's take a closer look at the known members of the DBV class and see what they have in common.

KUV 05134+261: Discovered by Grauer et al. (1989), it only has the discovery runs available. It is clearly multiperiodic, the pe- 
riods fall between $420 \mathrm{~s}$ and $770 \mathrm{~s}$, and the dominant period (upon discovery) is near $700 \mathrm{~s}$.

CBS 114: Discovered by Claver \& Winget, (1989) this star also has only the discovery data available. The dominant period is around $650 \mathrm{~s}$, with power present from about $300 \mathrm{~s}$ to over $700 \mathrm{~s}$. At magnitude 17, CBS 114 also has the present distinction of being the faintest DBV star.

PG1115+158: This star was a WET target during Spring of 1992, but the Fourier Transform (FT) refused to stabilize, defeating the longitudinal coverage capabilities of the WET. As a result, we do not have a proven mode identification, but Clemens et al. (1991) did the best they could with the data. Their analysis suggests several modes are present, which could be a set of semi-consecutive $\ell=1$ modes. Bradley (1993) assumed this identification to be correct, and derived a seismological mass near $0.62 \mathrm{M}_{\odot}$ and a helium layer mass of $10^{-4} \mathrm{M}_{\star}$ or less. Long term monitoring from a single site might tell us if the mode structure is inherently unstable, or if the fine structure splitting is near $12 \mathrm{~d}^{-1}$ or almost exactly $1 \mathrm{~d}^{-1}$.

PG 1351+489: A Spring 1995 WET run proves this star has other periods besides the ones at $489 \mathrm{~s}$ and $333 \mathrm{~s}$ found by Winget et al. (1987). This data set is under analysis, so it is too early to tell what the mode identification and seismological structure are.

PG 1456+103: This star is the first DBV discovered by Grauer et al. (1988). It has similar photometric properties to KUV 05134. I believe we will find it has a similar effective temperature and internal structure to KUV 05134 and GD 358.

GD 358: The subject of WET runs in May 1990 and May 1994, this star has a mass of $0.58 \mathrm{M}_{\odot}$ and a helium layer mass of $2 \times 10^{-6} \mathrm{M}_{\star}$, given the presently favored temperature of $25-26,000 \mathrm{~K}$ (Beauchamp et al. 1995 and Provencal et al. 1995). This mass determination is lower than given by Bradley \& Winget (1994) because the effective temperature determination is hotter now. More about this star later.

PG 1654+160: Not an official WET target yet, but several large glass data sets are available. They show the period of the dominant peak varies with time, as do the amplitudes. This star also has a red dwarf companion about $3^{\prime \prime}$ away, which could be a physical companion. If true, this star would not be a descendant of the IBWDs.

EC 20058-5234: The newest - and only southern hemisphere - DBV. This star has a number of short periods, and none is over 
300 s. All the other DBVs have all their periods longer than $300 \mathrm{~s}$, making EC 20058 an interesting complement. If we believe the temperature scaling of Clemens (1994) for the DAV stars, and apply it here, it would suggest that this star is the hottest DBV. Hopefully, an HST spectra will be available soon to tell us if this is the case.

\section{The DBV Stars According to GD 358}

In part because GD 358 is a bright large amplitude DBV, the May 1990 WET run revealed a wealth of pulsation modes (Winget et al. (1994), and the resultant seismological fit is the basis of our seismological understanding of the DBV stars. The data set is sufficiently rich that it forms the basis of several dissertations and Master's degree projects.

The effective temperature of GD 358 recently got updated; according to Beauchamp et al. (1995) it is, 25, 300 $\pm 300 \mathrm{~K}$ and $\log g=7.85$. Bradley \& Winget (1994) indicated their mass determination was temperature dependent. I did some additional modeling and find the mass is closer to $0.58 \mathrm{M}_{\odot}$ and the helium layer mass is essentially unchanged. Other values, such as the seismological parallax also changed; the best parallax distance is now about $45 \mathrm{pc}$, and $\log g=7.95$. Provencal et al. (1995) find a slightly hotter temperature of $\sim 27,000 \mathrm{~K}$. Depending on the carbon mass fraction in the core, I will probably be able to obtain decent fits for models between 0.58 and $0.55 \mathrm{M}_{\odot}$.

Winget et al. (1994) found asymmetries in the fine structure splitting. In all cases, the prograde mode splitting was larger, typically by 0.2 to $0.5 \mu \mathrm{Hz}$. Montgomery (1995) explored this idea in more detail by expanding on the work of Jones et al. (1989). Montgomery finds that a global magnetic field will not replicate the observed mode-to-mode asymmetries in detail. Either the simplified magnetic field geometries he uses are inadequate, or a magnetic field is not responsible for the frequency splitting asymmetries.

Two groups attacked the GD 358 progenitor problem. First, Dehner \& Kawaler (1995) considered the possibility that GD 358 evolved from a PG 1159 spectral type progenitor. They took Kawaler \& Bradley's (1994) He/C/O chemical composition profile for PG 1159-035 and let diffusion act. By $25,000 \mathrm{~K}$, the profile had a pure helium surface, a main $\mathrm{He} / \mathrm{C}$ transition zone at about $1.5 \times 10^{-6} \mathrm{M}_{\star}$ - as inferred by seismology - and a second $\mathrm{He} / \mathrm{C}$ transition zone down near $4 \times 10^{-3} \mathrm{M}_{\star}$. The total mass of helium in 
Dehner \& Kawaler's model is about $10^{-3.5} \mathrm{M}_{\star}$, just what Pelletier et al. (1986) found for the helium layer mass of the DQ white dwarfs. If diffusion can make the composition profile relax to nearly what one expects from diffusive equilibrium in the carbon "tail" extending towards the surface, then we have the suggestive possibility that PG 1159 stars evolve into something like GD 358, which then becomes a DQ star by about $12,000 \mathrm{~K}$. The only problem with this picture is the requirement that the PG 1159 stars have enough hydrogen present to make it turn into a DA star for the journey through the DB gap. The amount of hydrogen that can be present is small, because the star has to convectively mix the hydrogen into the helium layer at around $30,000 \mathrm{~K}$ for the star to become a DB. Further evolutionary calculations that track the diffusion and mixing of hydrogen should tell us if this picture for DB white dwarf evolution is correct.

Another possibility is that at least some DBV stars are descendants of the IBWDs. Nitta (1995) is constructing "hybrid" DB models that have a hot envelope surrounding a cooler core in an effort to mimic the coalesced product of an IBWD. Nitta will compare the pulsation properties of these models with the results of Bradley, Winget, \& Wood (1993) to determine the observable consequences. Assuming there is a difference in the predicted pulsation properties, we should be able to see if any DBV white dwarfs could have been sired by AM CVn systems. In this vein, PG $1654+160$ could be an important test object; if the red dwarf is a physical companion, then we would know it did not descend from an IBWD. If there is any difference between PG 1654's structure and the other DBV stars, this would provide - circumstantial - evidence that the oddball has some other progenitor, possibly an IBWD.

\section{Rating the DBV Stars as WET Targets}

Here, I consider the desirability of the DBV stars as WET targets. All of these stars are multiperiodic, even with single-site data, which makes them desirable from a seismological standpoint. However, except for GD 358, they are all magnitude 15.8 and below, which makes it difficult for us to get decent signal-to-noise data on them. This is a strike against the DBV stars and strong incentive to try and upgrade the WET instruments to reach fainter objects. In Table 1, I briefly list the pros and cons of each DBV as a WET target; in three cases, the star already has WET observations and I 
list this as a con, with the idea that we need to analyze the existing data before re-observing it.

TABLE 1: Names, Positions, and Magnitudes of the DBV Stars

\begin{tabular}{|c|c|c|}
\hline \multicolumn{2}{|c|}{$\begin{array}{ll}\text { NAME } & \text { PRO }\end{array}$} & \multirow{2}{*}{$\begin{array}{l}\text { CON } \\
\text { companion at } 15^{\prime \prime} \\
\text { no } T_{\text {eff }}\end{array}$} \\
\hline KUV $0513+26$ & Multimode & \\
\hline $\begin{array}{l}\text { CBS } 114 \\
\text { PG } 1115+158\end{array}$ & Founatorial & $\begin{array}{l}\text { Very Faint, No } T_{e} \\
\text { XCOV } 7 \text {, unstable }\end{array}$ \\
\hline$P G 1351+489$ & $\begin{array}{l}\text { Unique(?) mode structure, } \\
\text { has } T_{\text {eff }}\end{array}$ & $\begin{array}{l}\text { observed May } 199 \\
\text { (XCOV 12) }\end{array}$ \\
\hline PG $1456+103$ & Mod. Amplitude, & \\
\hline GD 358 & $\begin{array}{l}\text { Bright, } \\
\text { has seismological data }\end{array}$ & XCOV 5 s and 10 \\
\hline PG $1654+160$ & Equatorial, has $\mathrm{T}_{\text {eff }}$ & $\begin{array}{l}\text { M4 dwarf at } 3^{\prime \prime} \text {, } \\
\text { fairly rich field }\end{array}$ \\
\hline $20058-5$ & $\begin{array}{l}\text { ht, } \\
\text { rertones }\end{array}$ & $\begin{array}{l}\text { Southern Dec., } \\
{\text { no } T_{\text {eff }}}\end{array}$ \\
\hline
\end{tabular}

Note: All have multiperiodic behavior as a "pro" and faintness (except for GD 358) as a "con".

Having said all this, I suggest EC $20058-5234$ as the next DBV target for the WET. It will be very interesting to compare its structure with that of GD 358 to see if there is a fundamental structural difference or if EC 20058's effective temperature is so high that it is too close to the blue edge for long period modes to be excited - yet. By coincidence, the WET conclave picked EC 20058 as a target for the Fall 1996 WET run. I can't recommend looking at any other DBV stars with the WET until we see what PG $1351+489$ tells us. If we can extract sufficient seismological information from this star, then we have some hope of getting useful information out of PG $1456+103$ or PG1654+160, and either would make a promising candidate. I would give the nod to PG $1654+160$, because the red dwarf companion - if physically associated - would rule out an AM CVn system being the progenitor. A comparison of PG $1654+160$ to the other DBV stars may tell us if there is any seismological evidence for more than one class of progenitor for the DBV stars. 


\section{Rampant Speculations and Future Directions}

While it is possible that most or all of the cooler $\left(\mathrm{T}_{\text {eff }}<\right.$ $20,000 \mathrm{~K}) \mathrm{DA}$ white dwarfs are the spawn of hydrogen-rich planetary nebula nuclei, the picture is less certain for the DBV and DB white dwarfs. There are at least two possible progenitors of DB white dwarfs: the PG 1159 stars and the interacting binary white dwarfs. Dehner \& Kawaler (1995) show the seismological helium profile of PG 1159-035 can evolve into something similar to what Bradley \& Winget (1994) derive for GD 358, but they require two additional calculations before they can rest their case. First, they need add a small amount of hydrogen to the PG 1159-035 profile and show that the model will be a DA white dwarf in the DB gap, and then change to a DB white dwarf near $30,000 \mathrm{~K}$. Pelletier et al. (1986) shows the helium layer mass should be about $10^{-3.5} \mathrm{M}_{\star}$ in order to explain the "carbon pollution" trace abundances seen in helium rich white dwarfs below $15,000 \mathrm{~K}$. If the Dehner \& Kawaler calculations can be extended down to $12,000 \mathrm{~K}$ or below, they should be able to show the total helium layer mass of GD 358 is really about $10^{-3.5} M_{\star}$, consistent with the value of Pelletier et al. (1986). Doing this will remove the objection that the helium layer mass of GD 358 is too thin for the atmosphere to remain helium-rich below $15,000 \mathrm{~K}$.

Although the PG 1159 star - DB white dwarf link is the best studied, some DB white dwarfs should be descendants of IBWD systems, based on circumstantial evidence. As the name implies, the IBWD stars are already white dwarfs, there is no evidence for hydrogen in either star, the mass gaining star has an effective temperature at or above the red edge of the "DB gap", and the total mass of the two stars is less than the Chandrasekhar mass $\left(\sim 1.4 \mathrm{M}_{\odot}\right)$. All of these facts suggest that the IBWD stars should give rise to at least some DB white dwarfs, and possibly some DBV stars as well. Although there is no a priori reason to expect the structure of a DBV star with a PG 1159 star progenitor to be the same as a DBV star with an IBWD progenitor, only seismology has the chance to peer underneath the surface and find the potential thermal and structural clues. Our best hope here is to assume the IBWD descendants will become DBV stars; once we have seismologically determined structures for the DBV stars as a class, we can look to see if the structure of a few DBVs is systematically different from the rest. Unfortunately, this process depends on several assumptions, and I don't see a quick and easy way to settle the progenitor issue. 
I mentioned speculations earlier; here, I wish to suggest some ideas of what we will find for the DBV stars once the requisite observations and calculations are done. First, I believe that the mean period - effective temperature correlation Clemens (1994) found for the DAV white dwarfs is real, and further, I believe we will find it also applies to the DBV stars as well. Supposing this to be the case, I predict we will find that EC 20058-5234 is the hottest of the DBV stars, followed by PG $1351+489$. From the available data, PG $1115+158$ has the longest mean period of all the DBV stars, so I suggest it is the coolest. GD 358 and the remaining DBVs all have roughly the same mean period, so they probably all have about the same effective temperature. Except for EC 20058 and PG 1351, all of the DBVs with enough observations have significant amplitude variations with time for their main pulsation modes. If we accept the ordering of effective temperature given above, then the different amplitude variation behavior is probably telling something about mode selection. That is, as the partial ionization zone deepens, it becomes more able to shuffle pulsation energy from one mode to the other. Quantifying this behavior requires long term monitoring from a single site, which suffers from the vagaries of the weather and lunar phases. An examination of some GD 358 observations I took in 1992 suggest that significant amplitude changes occur on timescales of a month or less. I also looked into preliminary seismological analyses of other DBV stars. For the moment, I only ask "are models similar to what I used to fit the GD 358 pulsation spectrum consistent with the pulsation spectrums of other DBVs?". For the DBV stars with enough data, the answer seems to be "yes". However, I must caution that while this structure may be consistent with the available observations, $I$ have not yet done the work to rule out other possible fits. This will require a systematic exploration of a grid of DB models along with observational data of sufficient quality to allow unambiguous mode identification. I am sticking my neck out on all of these speculations, but at least they provide starting points for future work.

Finally, there are some needs we can address which will add much to our understanding of DBV stars. At present, our effective temperature determinations are uncertain by $2-3,000 \mathrm{~K}$, which means we cannot tell if there is even an instability strip for the DBVs. UV spectra from HST, along with the new model atmospheres of Beauchamp et al. (1995) and Provencal et al. (1995) should go a long way towards solving this problem. In addition to locating the instability strip, we will also be able to determine the red edge of 
the $\mathrm{DB}$ gap and determine how the DBA stars fit in the convective mixing picture for the onset of $\mathrm{DB}$ white dwarfs. If we can get the UV spectra, it would be valuable to determine the atmospheric parameters of the DB stars as a class to see if their mean mass is the same as for the DAs or not. While this probably won't settle the progenitor issue, it would shed light on homogeneity of the white dwarf formation process. Finally, determining the structure of all the DBV white dwarfs is critical. It will tell us if GD 358 is a freak; give us the mean mass of the DBV white dwarfs for comparison to the DAs and the nonpulsating DBs; and constrain the properties of DB progenitors.

Acknowledgements.

This research was supported by Los Alamos National Laboratory via a Director's Funded Postdoc.

\section{References}

Beauchamp, A., Wesemael, F., Bergeron, P., Saffer, R.A., \& Liebert, J. 1995, in Proc. 9th European Workshop on White Dwarf Stars, eds. D. Koester \& K. Werner (Berlin: Springer), 108

Bradley P. A., 1993, Ph. D. Thesis, Univ. Texas.

Bradley P. A., Winget D. E., 1994, ApJ, 430, 850

Bradley P. A., Winget D. E., Wood M. A., 1993, ApJ 406, 661

Clemens J. C. et al., 1993. In Barstow M. A. (ed.) White Dwarfs: Advances in Observations and Theory. Kluwer, Dordrecht, p. 515

Dehner, B.T., \& Kawaler, S.D. 1995, ApJ, 445, L141

Grauer, A.D., Bond, H.E., Green R.F., \& Liebert, J. 1988, AJ, 95, 879

Grauer, A.D., Wegner, G. \& Liebert, J. 1989, AJ, 98, 2221

Montgomery, M. H., 1995, M. S. Thesis, Univ. Texas.

Nather R. E., Winget D. E., Clemens J. C., Hansen C. J., Hine B. P., 1990, ApJ 361, 309

O'Donoghue, D. 1995, in proc. 9th European Workshop on White Dwarfs, eds. D. Koester \& K. Werner, 297

Pelletier, G., Fontaine, G., Wesemael, F., Michaud, G., \& Wegner, G. 1986, ApJ, 307, 242

Provencal, J.L., Shipman, H.L., Thejll, P., Vennes, S., \& Bradley, P.A. 1995, ApJ, submitted

Winget, D.E. \& Claver, C.F. 1989, in IAU Colloq. 114, White Dwarfs, ed. G. Wegner, (Berlin: Springer), 290

Winget, D.E., Nather, R.E., \& Hill, J.A. 1987, ApJ, 316, 305 
Winget, D.E., Robinson, E.L., Nather, R.E., \& Balachandran, S. 1984, ApJ, 279, L15

Winget, D. E., et al. 1994, ApJ, 430, 839 